\title{
Se souvenir des belles choses
}

\author{
Y. Panis \\ () Springer-Verlag 2009
}

C'était il y a bien longtemps. À l'époque, les Journées francophones de pathologie digestive ouvraient largement leurs portes aux chirurgiens digestifs. La pathologie hépatobiliaire et pancréatique, de même que colorectale, se prêtait en effet très bien à une approche médicochirurgicale. On avait même envie de dire que l'évolution de nos métiers faisait qu'il semblait très difficile, pour ne pas dire impossible, que les hépatogastroentérologues et les chirurgiens envisagent de travailler chacun de leur côté. Ces JFPD (comme on disait à l'époque) étaient le lieu privilégié de la formalisation, à l'échelon national, de cette communauté. C'était aussi le lieu de rencontres amicales (et de soirées un peu arrosées...) avec des collègues de toute la France que bien souvent nous n'avions pas revus depuis le congrès précédent!

Cette grand-messe, pour nous chirurgiens, était un rendez-vous marqué d'emblée sur le nouvel agenda Exacompta acheté $(4 €)$ le $1^{\text {er }}$ janvier de l'année ! C'était tout simplement impensable d'imaginer que nous allions rater cet événement scientifico-médico-chirurgicalo-amical. Inimaginable !

Nommé interne en 1985, je me souviens de mes premières JFPD. C'était à Lille je crois. Jeune con plein d'ambition, j'avais un abstract accepté au congrès sur mon DEA. Pas encore le droit de causer comme les grands. Mais je m'étais, non sans une certaine émotion, présenté le premier jour à la séance plénière pour aller écouter les grands fauves de la spécialité. Je me souviens y avoir croisé J. Belghiti, révisant son topo, dans un des couloirs du palais des Congrès. J'avais envie un jour d'être à sa place...

Depuis 1988, je n'ai pas raté une seule fois les JFPD. Vingt et un congrès de suite! Sans aucune infidélité !

Cette année ? Je n'y suis pas allé. Pour quelle raison? Une invitation à un congrès international ? Un enterrement ? Une manifestation contre une réforme de l'hôpital ? Un mariage ? Non. Rien de tout ça. Je n'y suis pas allé, car je n'avais pas envie. La raison? Elle est très simple : je n'y

Y. Panis $(\bowtie)$

Service de chirurgie colorectale, pôle des maladies

de l'appareil digestif (PMAD), hôpital Beaujon, 100, boulevard

du Général-Leclerc, F-92118 Clichy cedex, France

e-mail : yves.panis@bjn.aphp.fr suis pas allé, car je ne me retrouve pas dans ce nouveau congrès. Il paraît que ça s'appelle maintenant les JFHOD, comme Journées francophones d'hépatogastroentérologie et d'oncologie digestive. Je n'ai rien à dire sur le titre... Et le problème n'est évidemment pas le titre. Le problème, pour ne rien vous cacher, c'est qu'avant, je me précipitais sur le programme, et je regardais les sessions « chirurgicales » en premier. Ou plus exactement, les sessions de la SFCD (Société française de chirurgie digestive) et de l'ACHBT (Association de chirurgie hépatobiliaire et de transplantation). Ah! Pas par mépris pour les gastroentérologues ! Bien au contraire. Mais seulement, je savais que j'y retrouverais mes copains. Qu'il y aurait au minimum deux matinées pour la SFCD, deux de l'ACHBT, souvent aussi un (voire deux) symposium organisé par les chirurgiens, la séance plénière et puis un bon repas avec mes potes médecins. C'était la belle vie quoi ! En plus, j'avais eu la chance de les côtoyer de près, moi, ces grands fauves du versant médical de la spécialité. Au titre de secrétaire général de la SFCD, je suis resté en effet cinq ans au conseil scientifique des JFPD, avec Étienne Dorval en grand maître de cérémonie. C'était une réunion annuelle où l'ensemble des membres discutait du programme scientifique, choisissait les communications (parmi les meilleures déjà sélectionnées par d'autres) qui devaient avoir l'insigne honneur d'être retenues en séance plénière. Les réunions étaient parfois un peu houleuses. Je me suis quelquefois battu pour défendre les abstracts « chirurgicaux ». Mais le grand débat qui a agité nos réunions du conseil scientifique était ce fameux pourcentage d'acceptation des abstracts. Pour vous la faire courte, les abstracts du tronc commun, c'est-à-dire de toute l'hépatogastroentérologie (sauf l'AFEF ou Association française pour l'étude du foie), étaient (si j'ai bonne mémoire) de seulement $15 \%$. Alors que nous, chirurgiens (et peut-être l'AFEF aussi mais je ne sais plus), avions des taux scandaleux d'environ 30 à $50 \%$. Il était donc facile de montrer du doigt les vilains petits canards qu'étaient ces trois sociétés savantes qui, elles, avaient le droit ancestral d'avoir des sessions séparées : l'AFEF, l'ACHBT et notre chère SFCD.

Pendant toutes ces années, j'ai néanmoins réussi, avec l'aide de mes collègues de ces deux sociétés, à défendre 
auprès d'Étienne Dorval notre particularité avec l'argument principal que cette stratégie permettait d'amener au congrès des chirurgiens digestifs habituellement peu enthousiastes à l'idée d'aller traîner dans les congrès médicaux. Et finalement, qu'importe ce taux de sélection très élevé. Et pourquoi parler d'injustice, puisque nous n'étions finalement qu'une «annexe» de ce prestigieux congrès des JFPD. Je dois remercier ici Étienne Dorval qui nous a toujours soutenus pour maintenir cette situation, contre l'avis parfois violent de certains de ses collègues.

Évidemment, les bonnes choses ont toujours une fin. Et le changement de bureau de la SNFGE et de son secrétaire général (avec l'arrivée de Guillaume Cadiot qui est un ami et que je respecte) a changé la donne. Aujourd'hui, ce nouveau bureau a décidé de changer les règles, c'est son droit, avec la disparition de cette particularité pour ces trois sociétés savantes, leurs travaux étant désormais sélectionnés dans un seul tronc commun. Le résultat est qu'il y a, à l'évidence, une parfaite égalité entre les différents abstracts.

Le prix à payer : la quasi-disparition des chirurgiens dans les couloirs et les salles. En tout cas, une analyse extrêmement scientifique portant sur dix copains est sans appel : aucun n'y a mis cette année les pieds.

La raison principale est le manque de lisibilité des topos chirurgicaux, qui sont souvent mélangés à des topos médicaux, certes passionnants, mais qui ne déplacent pas les foules chirurgicales, bien au contraire !

Quelques exemples parmi d'autres : une session commune entre la SFED, la SNFGE... et notre chère SFCD : sur huit présentations orales... une seule chirurgicale (et encore... sur notes) ! Pensez-vous vraiment qu'un chirurgien va venir écouter cette session pour un seul papier chirurgical ? La réponse est évidemment non. Pareil le même jour pour une autre session de cancérologie, sous "l'égide » de la SFCD en titre: deux présentations chirurgicales sur neuf ! Et il y a pire encore : une autre session de cancérologie en partenariat avec la SFCD : sur sept présentations cancérologiques, aucune chirurgicale ! Bon, il faut être honnête. Le vendredi, nous avions un symposium de la SFCD sur le cancer de l'œsophage et une session intitulée « chirurgie ». Enfin, sur huit présentations, quatre vraiment de chirurgie. Tiens ! Un autre exemple, une session sur le reflux, avec la SFCD à nouveau dans le titre. Eh bien, sur neuf présentations orales... une seule chirurgicale!
Le but de mon propos n'est pas ici de critiquer la qualité des papiers médicaux présentés et de «râler » en disant qu'un grand nombre de papiers chirurgicaux a été refusé pour des présentations orales. Non, le but ici est de penser aux collègues chirurgiens que je connais bien. La grande majorité d'entre eux venait à ces journées pour aller dans des sessions chirurgicales, aux séances plénières et aux symposia médicochirurgicaux. Aujourd'hui, il est difficile de penser que les chirurgiens seront assez motivés pour assister à une séance complète de neuf présentations afin d'écouter seulement une seule présentation chirurgicale! Quel gastroentérologue irait écouter huit papiers chirurgicaux pour une seule présentation médicale ? Enfin, quand on analyse le volume global de papiers chirurgicaux, on voit clairement une diminution, puisqu'au final, je n'ai retrouvé dans le programme qu'une seule session intitulée chirurgie contre trois ou quatre, les années précédentes.

Tout cela est évidemment le choix du nouveau Bureau, et étant légitimiste de nature, je le respecte totalement. Néanmoins, je pense que cette voie n'est pas la bonne ; car chaque jour dans nos hôpitaux, nous travaillons en très étroite collaboration entre gastroentérologues et chirurgiens. La grand-messe annuelle de l'hépatogastroentérologie devrait refléter cet état de fait. Ce n'est pas le cas aujourd'hui et c'est bien dommage. Finalement, qu'auraient à perdre les gastroentérologues si, dans des salles du congrès, se déroulaient deux ou trois sessions strictement chirurgicales ? Rien, évidemment. Ils auraient tout à y gagner. Non seulement certains médecins pourraient venir écouter certains papiers qui les intéressent, mais aussi les chirurgiens seraient plus nombreux à écouter les papiers médicaux, évidemment très enrichissants pour nous. N'étant plus aux affaires de la SFCD, je n'ai évidemment aucun pouvoir à proposer quoi que ce soit! Mais je pense qu'une solution pourrait être, par exemple, à la SFCD, à l'ACHBT, voire à l'AFC (Association française de chirurgie qui est notre grand-messe) de proposer à nos amis hépatogastroentérologues de participer et d'organiser des sessions médicales, comme nous le faisions à l'époque aux JFPD. De même, aux JFHOD ? Des sessions chirurgicales seraient organisées parallèlement au programme médical. Je pense sincèrement que tout le monde y gagnerait. Les chirurgiens ont besoin des gastroentérologues... Et réciproquement ! Et puis, il faut être honnête : à Midi, Porte Maillot, les déjeuners avec mes copains gastros me manquent beaucoup ! 